\title{
A Novel Three-Dimensional Culture System of Polarized Epithelial Cells to Study Endometrial Carcinogenesis
}

\author{
Núria Eritja, David Llobet, Mónica Domingo, \\ Maria Santacana, Andree Yeramian, \\ Xavier Matias-Guiu, and Xavi Dolcet \\ From the Oncologic Pathology Group, Hospital Universitari \\ Arnau de Vilanova, Institut de Recerca Biomèdica de Lleida. \\ IRBLleida, University of Lleida, Lleida, Spain
}

Development of three-dimensional (3D) cultures that mimic in vivo tissue organization has a pivotal role in the investigation of the involvement of cell adhesion and polarity genes in the pathogenesis of epithelial cancers. Here we describe a novel 3D culture model with primary mouse endometrial epithelial cells. In this model, isolated endometrial epithelial cells develop single-lumened, polarized glandular structures resembling those observed in endometrial tissue. Our in vitro 3D culture model of endometrial glands requires the use of serum-free defined medium with only epidermal growth factor and insulin as growth supplements and 3\% Matrigel as reconstituted extracellular matrix. Under these culture conditions, glands of epithelial cells displaying typical apicobasal polarity and proper positioning of tight and adherent junctions are formed by hollowing as early as 7 to 8 days in culture. Addition of the phosphatidylinositol 3-kinase inhibitor LY294002 completely inhibits bromodeoxyuridine incorporation and cyclinD1 expression, confirming that in vitro growth of endometrial glands depends on phosphatidylinositol 3-kinase/Akt signaling. To prove that our culture method is a good model to study endometrial carcinogenesis, we knocked down E-cadherin or phosphatase and tensin homolog expression by lentivirus-delivered short hairpin RNAs. Down-regulation of E-cadherin resulted in complete loss of epithelial cell polarity and glandular formation, whereas phosphatase and tensin homolog down-regulation resulted in increased proliferation of glandular epithelial cells. These properties indicate that our 3D culture model is suitable to study the effect of growth factors, drugs, and gene alterations in endometrial carcinogenesis and to study normal endometrial biology/physiology. (AmJ Pathol 2010, 176:2722-2731; DOI: 10.2353/ajpath.2010.090974)

In the endometrium and other glandular tissues, epithelial cells interact with neighboring cells and extracellular matrix to develop well organized and well polarized threedimensional glands. Increasing evidence indicates that appropriate three-dimensional (3D) organization is critical for tissue homeostasis. ${ }^{1}$ Maintenance of cell polarity and cell-to-cell and cell-to-matrix adhesion plays a pivotal role in the regulation of glandular homeostasis and epithelial cell proliferation, differentiation, and survival. During carcinogenesis, disruption of glandular architecture and loss of epithelial polarity leads to increased tumorigenic potential. ${ }^{2-5}$ Alterations in genes that control formation of cell contacts result in loss of cell polarity and cause increased proliferation and migration capabilities, two critical processes for development of malignant transformation.

Proteins that participate in formation of cell-to-cell contacts are potent tumor suppressors, and they have been found to be deregulated in cancer. ${ }^{6}$ Among the genes encoding adhesion or polarity molecules, epithelial cadherin (E-cadherin) has been recognized as a critical pro-

Supported by grants from Fondo de Investigaciones Sanitarias (FIS070304, FIS070276), from Ministerio de Ciencia e Innovación (SAF2002-10529-E, SAF2004-05250), and from Xarxa Temática de la Generalitat de Catalunya (2004XT00090), La Asociación Española Contra el Cáncer, Catalunya contra el cancer and programa de intensificación de la investigación, Instituto Carlos III. N.E. holds a fellowship from Fundación Alicia Cuello de Merigó and a predoctoral fellowship from Fondo de Investigaciones Sanitarias (FIS) (FI08/0012). D.L. holds a predoctoral fellowship from FIS, Ministerio de Sanidad y Consumo (FI05/00191). M.S. is supported by Red Tematica de Investigacion Cooperativa en Cancer (RD06/0020/1034). A. Y. holds a Juan de la Cierva Fellowship. X.D. holds a Miguel Servet fellowship from FIS, Ministerio de Sanidad y Consumo (CP05/00028).

Accepted for publication January 26, 2010

Supplemental material for this article can be found on http://ajp. amjpathol.org.

Address reprint requests to Xavi Dolcet, Ph.D., or Xavier Matias-Guiu, Ph.D., Laboratori de Recerca Biomèdica, Hospital Arnau de Vilanova, Av Rovira Roure, 80, 25198 Lleida, Spain. E-mail: dolcet@cmb.udl.cat and xmatias@arnau.scs.es. 
tein for adherent junction formation and maintenance of cell polarity. Genetic or epigenetic alterations leading to a decrease or loss of E-cadherin expression have been identified in a wide variety of malignant epithelial tumors, including those from the breast ${ }^{7,8}$ or the endometrium. ${ }^{9-11}$ Loss of E-cadherin expression results in loss of polarity, increased migration, and development of epithelial-to-mesenchymal transition. ${ }^{12,13}$

Development of 3D culture systems has been critical to study the role of cell adhesion and polarity genes in the pathogenesis of cancer. 3D culture systems are important tools to advance in knowledge of the mechanisms involved in the development and progression of cancer. 3D cultures provide important information at several levels: i) the mechanisms of glandular lumen formation and maintenance; ii) the role of cancer genes on cell polarity; and iii) the role of cell-to-cell and cell-to matrix contacts in carcinogenesis. Moreover, the 3D microenvironment may alter intracellular cell signaling, which ultimately triggers the cellular response to different extracellular stimuli.

3D cultures from epithelial cells were first established from different epithelial tissues or cell lines using collagen-based matrices. ${ }^{14-18}$ However, over the last few years, the use of epithelial cells derived from breast tissue or breast epithelial cell lines has led to the development of 3D cultures. ${ }^{19-24}$ Among all of the methods to establish $3 \mathrm{D}$ cultures, the ones using a reconstituted basement membrane (rBM) derived from EngelbrethHolm-Swarm tumors as extracellular matrix have been the first choice for breast and other glandular epithelial tissues. There are three main systems to develop 3D cultures from epithelial cells using rBM. ${ }^{20,25}$ In the first method, cells are completely embedded in rBM, in the second method, cells are seeded in an overlay of rBM and cultured in medium containing $2 \% \mathrm{rBM}$, and in the third method cells are seeded on polyacrylamide-linked rBM with overlaying of diluted rBM. In addition to the appropriate extracellular matrix, epithelial cells also require growth supplements in culture medium to develop well structured acini, spheroids, or glandular structures. The type of media, serum, or supplements varies, depending on cell type and requires optimization for each particular case.

Here, we describe a novel 3D culture system of primary mouse endometrial epithelial cells. This culture system incorporates some innovations and advantages over other 3D culture systems. 3D endometrial glandular structures are developed in serum-free, defined medium containing only insulin and epidermal growth factor (EGF) as growth factors. Moreover, endometrial glandular structures are developed in medium containing only $3 \%$ rBM without the need for an underlying bed of gelled matrix. Endometrial cells can be easily infected with lentiviruses before the setup of 3D cultures. Using this approach, we demonstrate that E-cadherin knockdown results in complete disruption of endometrial glands. To further evaluate our 3D culture as a good tool for studies of key alterations found in endometrial carcinogenesis, we have analyzed the effects of phosphatase and tensin homolog (PTEN) short hairpin RNA (shRNA) on epithelial cell pro- liferation. PTEN knockdown results in increased expression of cyclinD1 and increased proliferation. These properties make our 3D culture suitable for studies of the effects of growth factors, drugs, and candidate genes involved in morphogenesis and homeostasis of endometrial tissue.

\section{Materials and Methods}

\section{Reagents and Antibodies}

Matrigel (rBM) was purchased from BD Biosciences (San Jose, CA). Epidermal growth factor was from SigmaAldrich (St. Louis, MO), and insulin-transferrin-sodium selenite (ITS) supplement was from Invitrogen (Carlsbad, $\mathrm{CA}$ ). Antibodies to E-cadherin, $\beta$-catenin, GM130, and total extracellular signal-regulated kinase (ERK) were from BD Biosciences; ZO-1 was from Zymed Laboratories (South San Francisco, CA); bisBenzimide H 33342 trihydrochloride (Hoechst), rhodamine conjugated-phalloidin, and antibodies to laminin and tubulin were from Sigma-Aldrich, and anti-cytokeratin was from Abcam (Cambridge, UK). Alexa Fluor anti-rabbit and anti-mouse antibodies were from Invitrogen. Phospho-Akt, phosphoglycogen synthase kinase 3 (GSK3), and phospho-ERK antibodies were from Cell Signaling Technology (Beverly, MA). Antibody to cyclinD1 and total Akt were from Santa Cruz Biotechnology (Santa Cruz, CA), Peroxidase-conjugated anti-mouse and anti-rabbit antibodies were from Jackson ImmunoResearch Europe Ltd. (Suffolk, UK). All other regents were obtained from Sigma-Aldrich unless specified.

\section{Isolation of Mouse Endometrial Epithelial Cells}

C57BL6 mice used to isolate endometrial cells were maintained in temperature- and light-controlled conditions and fed ad libitum. All experimental procedures were approved by the Institutional Animal Care Committee of IRBLleida. Isolation of mouse epithelial cells was performed as described previously with some modifications. ${ }^{26}$ Mice were sacrificed by cervical dislocation, and uterine horns were dissected from 3- to 4-week-old C57BL6 mice. Uteri were washed with Hanks' balanced salt solution and chopped in 3- to 4-mm-length fragments. Uterine fragments were digested with $1 \%$ trypsin (Invitrogen) in Hanks' balanced salt solution (Invitrogen) for 1 hour at $4^{\circ} \mathrm{C}$ and 45 minutes at room temperature. Trypsin digestion was stopped by addition of Dulbecco's modified Eagle's medium (DMEM) containing 10\% fetal bovine serum (Invitrogen). After trypsin digestion, epithelial sheets were squeezed out of the uterine pieces by applying gentle pressure with the edge of a razor blade. Epithelial sheets were washed twice with PBS and resuspended in $1 \mathrm{ml}$ of DMEM/F12 (Invitrogen) supplemented with $1 \mathrm{mmol} / \mathrm{L}$ HEPES (Sigma-Aldrich), 1\% penicillin/ streptomycin (Sigma-Aldrich), and Fungizone (Invitrogen) (basal medium). Epithelial sheets were mechanically disrupted in basal medium by pipetting 50 times through a 1-ml tip, until clumps of cells are observed 
under the microscope. Any attempt to individualize cells results in a dramatic decrease in cell viability. Cells were diluted in basal medium containing $2 \%$ dextran-coated charcoal-stripped serum (HyClone Laboratories, Logan, UT) and plated into culture dishes (BD Falcon, Bedford, MA). Cells were cultured for 24 hours in an incubator at $37^{\circ} \mathrm{C}$ with saturating humidity and $5 \% \mathrm{CO}_{2}$.

\section{Establishment of 3D Cultures}

Twenty-four hours after plating in plastic, cells were washed with Hanks' balanced salt solution and incubated with trypsin/EDTA solution (Sigma-Aldrich) for 5 minutes at $37^{\circ} \mathrm{C}$. Trypsin digestion was stopped by addition of DMEM/10\% fetal bovine serum. Cells were collected in $15-\mathrm{ml}$ conical tubes and centrifuged at $1000 \mathrm{rpm}$ for 3 minutes, resuspended in Hanks' balanced salt solution, and disrupted by pipetting 15 to 20 times through a 1-ml tip until clumps of two to eight cells were obtained. Any attempt to further dissociate clumps to individualize cells resulted in a dramatic decrease in cell viability. Cells were centrifuged at $1000 \mathrm{rpm}$ for 3 minutes and diluted in basal medium containing 3\% Matrigel to obtain $4 \times 10^{4}$ cell clumps/ml. Twenty-four hours after plating, medium was replaced by basal medium supplemented with 5 $\mathrm{ng} / \mathrm{ml}$ EGF and a 1:100 dilution of ITS supplement and $3 \%$ fresh Matrigel (BIE medium). Medium was replaced every 2 to 3 days.

\section{Immunofluorescence}

For Western blotting cells were plated in a volume of 200 $\mu \mathrm{l}$ in 24-well plates (BD Biosciences). For immunofluorescence and bromodeoxyuridine experiments cells were seeded in a volume of $40 \mu \mathrm{l} /$ well in 96-well plates (black with microclear bottom, Greiner Bio-One, Longwood, FL). $3 D$ cultures were fixed with formalin for 5 minutes at room temperature and washed twice with PBS. Depending on the primary antibody, cells were permeabilized with $0.2 \%$ Triton X-100 in PBS for 10 minutes (indicated as T) or permeabilized with 100\% methanol for 2 minutes (indicated as M). Next, cultures were incubated overnight at $4^{\circ} \mathrm{C}$ with the indicated dilutions of antibodies: anti-Ecadherin, 1:200 (T); anti-ZO-1, 1:100 (T); anti-cytokeratin, 1:500 $(\mathrm{T})$; anti-laminin, 1:500 $(\mathrm{T})$; rhodamine-conjugated phalloidin, 1:500 (T), anti- $\beta$-catenin, 1:200 (M); or antiGM130, 1:100 (M). After 1 day, cells were washed twice with PBS and incubated with PBS containing $5 \mu \mathrm{g} / \mathrm{ml}$ Hoechst dye and a 1:500 dilution of Alexa Fluor secondary anti-mouse or anti-rabbit antibodies for 2 hours at room temperature. For double immunofluorescence staining, cells were incubated with the second round of primary and secondary antibodies. Note that in all double immunofluorescence stains, first and second primary antibodies were from different isotopes.

For immunofluorescence staining on mouse endometrium, uterine tissue was dissected in PBS, chopped in 3to 4-mm pieces and fixed with formol or methanol for 30 minutes at room temperature. Uterine pieces were embedded in Tissue-Tek and were cut in 10- $\mu \mathrm{m}$ slices using a cryostat. Cryostat sections were incubated with primary antibodies overnight at $4^{\circ} \mathrm{C}$ followed by a 1-hour incubation at room temperature with Alexa Fluor-labeled secondary antibodies.

Immunofluorescence staining was visualized and analyzed using a confocal microscope (Olympus, Tokyo, Japan). Images, 3D reconstruction, and video editing of confocal images were performed using FluoView software (Olympus).

\section{Bromodeoxyuridine Incorporation}

The bromodeoxyuridine protocol was performed as described previously with minor modifications. ${ }^{27} 3 \mathrm{D}$ cultures were incubated with $3 \mathrm{ng} / \mathrm{ml}$ 5-bromodeoxyuridine (BrdU) (Sigma-Aldrich) for 15 hours and then fixed with $4 \%$ paraformaldehyde. After DNA denaturing with $2 \mathrm{~mol} / \mathrm{L}$ $\mathrm{HCl}$ for 30 minutes and neutralization with $0.1 \mathrm{~mol} / \mathrm{L}$ $\mathrm{Na}_{2} \mathrm{~B}_{4} \mathrm{O}_{7}(\mathrm{pH}$ 8.5) for 2 minutes, cells were blocked in PBS solution containing $5 \%$ horse serum, $5 \%$ fetal bovine serum, $0.2 \%$ glycine, and $0.1 \%$ Triton $\mathrm{X}-100$ for 1 hour. Subsequently, cells were subjected to indirect immunofluorescence with a mouse 1:100 dilution of anti-BrdU monoclonal antibody (DAKO, Carpenteria, CA), and Alexa Fluor-conjugated anti-mouse secondary antibody. Nuclei were counterstained with $5 \mu \mathrm{g} / \mathrm{ml}$ Hoechst 33258, and cells were visualized under a confocal microscope. BrdU-positive nuclei were scored and divided by the total number of cells (visualized by Hoechst staining). The results are expressed as a percentage of BrdU-positive cells.

\section{Lentiviral Production and Infection}

Oligonucleotides to produce plasmid-based shRNA were cloned into the FSV vector using Agel-BamHI restriction sites. shRNA target sequences were as follows: PTEN, 5'-ATATAGGTCAAGTCTAAGTCG-3'; E-cadherin-1, 5'TGGTTCAGATCAAATCCAACA-3'; and E-cadherin-2, 5'TGCCAACTGGCTGGAGATTAA-3'. Lentiviral particles were produced as described previously with some modifications. $^{28}$ 293T human embryonic kidney cells were cotransfected by the polyethylenimine method with the virion packaging elements psPAX2 and PMD2G and the shRNA-producing vector FSV. 293T cells were allowed to produce lentiviral particles during 3 to 4 days in same culture medium used for endometrial cell lines and explants. Culture medium was collected, centrifuged for 5 minutes at $1000 \mathrm{rpm}$, and filtered through a $0.45-\mu \mathrm{m}$ filter (Millipore Corporation, Billerica, MA). The medium was concentrated by centrifugation through 100,000 MWCO Vivaspin 20 columns (Sartorious Stedium Biotech, Aubagne, France). Freshly isolated or thawed mouse endometrial cells were diluted in DMEM/F12 plus 2\% DCC-S as described above and plated into 24-well plastic dishes. Two to 3 hours after plating 10 to $20 \mu \mathrm{l}$ of concentrated lentiviral particles plus $8 \mu \mathrm{g} / \mathrm{ml}$ of hexadimethrine bromide (Polybrene; SigmaAldrich) were added to the cultures and incubated for 24 hours. After this infection period cells were processed to establish 3D cultures as described above. 


\section{Western Blot Analysis}

Endometrial 3D cultures were stimulated for the indicated periods of time, washed with cold PBS, and lysed with lysis buffer (2\% SDS, 125 mmol/L Tris- $\mathrm{HCl}, \mathrm{pH}$ 6.8). Relative protein concentrations were determined by loading on an $8 \%$ acrylamide gel, transferring to polyvinylidene difluoride membranes (Millipore Corporation), and blotting with antitubulin antibody. Band density was determined using Quantity One software (Bio-Rad Laboratories, Richmond, CA). Equal amounts of proteins were subjected to SDSpolyacrylamide gel electrophoresis and transferred to polyvinylidene difluoride membranes. Nonspecific binding was blocked by incubation with TBST $(20 \mathrm{mmol} / \mathrm{L}$ Tris- $\mathrm{HCl}, \mathrm{pH}$ 7.4, $150 \mathrm{mmol} / \mathrm{L} \mathrm{NaCl}$, and 0.1\% Tween 20) plus 5\% nonfat milk. Membranes were incubated with the primary antibodies overnight at $4^{\circ} \mathrm{C}$ followed by a 1-hour incubation with secondary antibody (1:10,000 in TBST). Signal was detected with ECL Advance (Amersham-Pharmacia, Little Chalfont, Buckinghamshire, UK).

\section{Results}

\section{Isolation of Endometrial Epithelial Cells from Mouse Uteri}

We first established a protocol to isolate endometrial epithelial cells from mouse uterus. As described in the Materials and Methods, we used a highly concentrated trypsin digestion to detach luminal epithelial sheets from the rest of uterine tissue. This procedure allows the separation of a tube containing epithelial cells from the rest of uterus containing mainly stromal cells (Figure 1A). The epithelial cell tubes were mechanically disrupted to obtain small cell clumps, which were plated into culture dishes and grown as two-dimensional (2D) monolayer cultures. Epithelial cell clumps grew clonally and displayed typical epithelial cobblestone morphology, whereas the stromal fraction showed typical fibroblastic morphology (Figure 1A). To ensure that epithelial cells were properly isolated, we performed immunostaining with different epithelial cell markers. Epithelial cells were positive for cytokeratin, E-cadherin, and $\beta$-catenin staining but negative for vimentin, a specific intermediate filament present in stromal cells (Figure 1B). As expected, cytokeratin staining was cytoplasmic, and both E-cadherin and $\beta$-catenin staining were confined to cytoplasmic membrane.

\section{Endometrial Epithelial Cells Develop 3D Glandular Structures in Serum-Free Defined Medium Containing Insulin and EGF}

Next, we developed a protocol to establish a polarized epithelial cell culture from endometrial cells grown in 2D cultures. Establishment of 3D cultures of epithelial cells requires a basement membrane and different growth supplements to drive the development of polarized glandular structures. Most 3D cultures of epithelial cells from different origins require specific growth factors and sup-
A
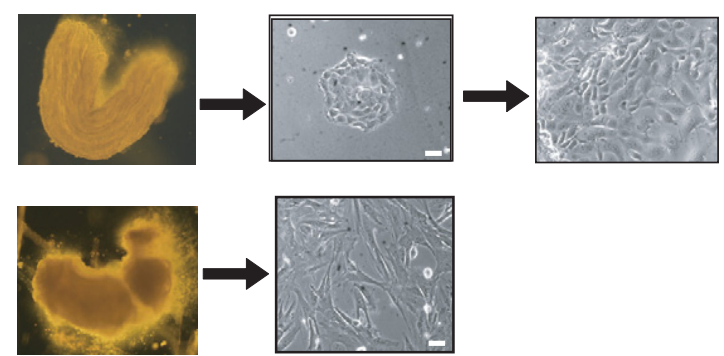

B

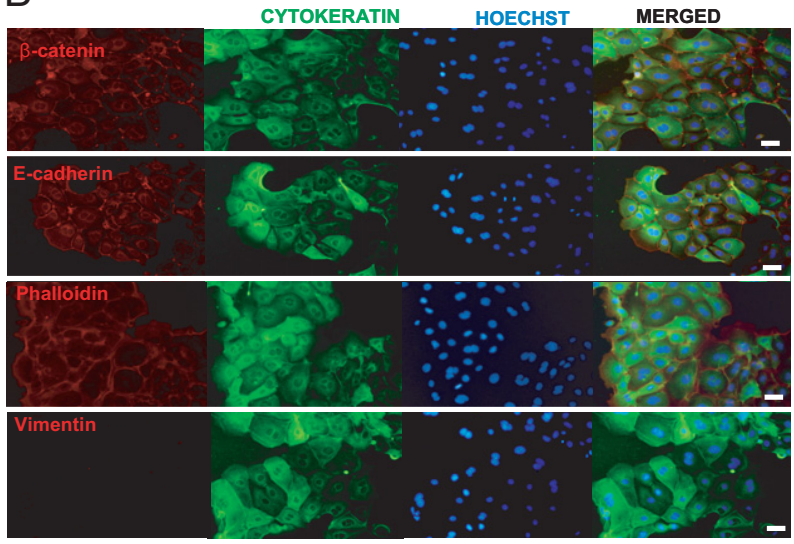

Figure 1. Isolation of epithelial and stromal endometrial cells from mouse uterus. A, left: representative images of the epithelial sheet (top) and the rest of the uterine horn fragment (bottom) after digestion with trypsin and separation of two parts. Right, representative images of isolated epithelial and stromal cells cultured in 2D monolayer cells for 24 hours. B: Double immunofluorescence showing positive labeling of 2D monolayer epithelial cells with cytokeratin, E-cadherin, $\beta$-catenin, and phalloidin antibodies and negative staining for vimentin. In all immunofluorescence experiments cells were counterstained with Hoechst dye. White scale bar $=20 \mu \mathrm{m}$.

plements such as insulin (usually added as part of the ITS supplement), EGF, hydrocortisone, and different amounts of serum. To set up 3D cultures from cells growing in monolayer, epithelial cells were trypsinized, washed, and resuspended in DMEM/F12 containing different combinations of growth supplements plus different concentrations of Matrigel. We first used DMEM/F12 containing a constant concentration of EGF and ITS plus increasing percentages of serum and concentrations of Matrigel. Medium containing as low as 1\% fetal bovine serum resulted in exaggerated growth of cells, which failed to acquire 3D polarity as assessed by E-cadherin immunostaining and phase-contrast morphology (Supplemental Figure S1, see http://ajp. amjpathol.org). In parallel experiments, cells were cultured in medium containing EGF plus ITS and increasing concentrations of Matrigel $(1,3,25$, and $50 \%$ ) diluted in culture medium. We set a percentage of $3 \%$ as the minimal concentration of Matrigel to obtain glandular-like structures. Lower concentrations (1\%) did not support glandular growth (Supplemental Figure S2, see http://ajp.amjpathol. org). Although higher concentrations of Matrigel led to gland formation, immunostaining of glands with some antibodies was impaired, probably because of a limited capability of antibodies to penetrate through a dense Matrigel matrix (Supplemental Figure S2, see http://ajp.amjpathol.org). Of note, endometrial epithelial 
A

BAS

B H

$B E$

B I

B H E

B H I

B HIE

B I E
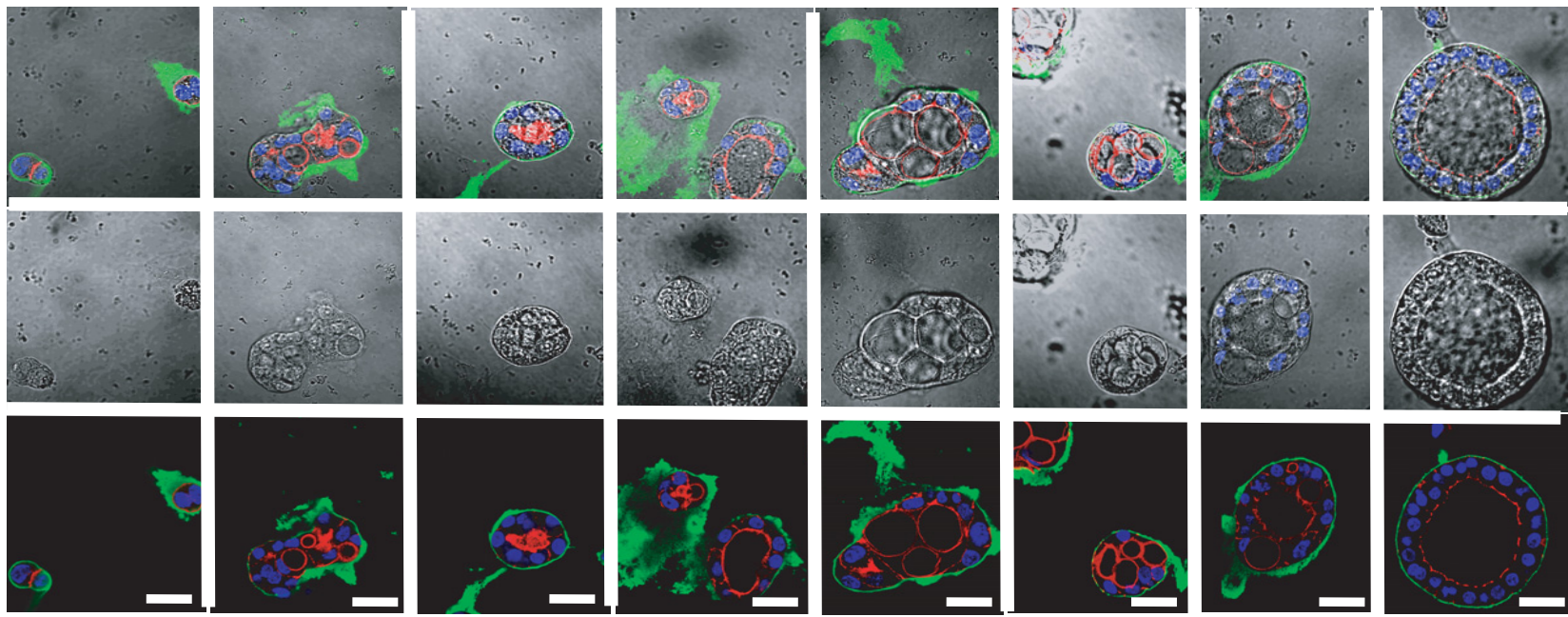

B

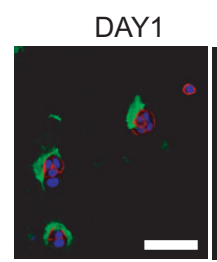

DAY2

DAY3
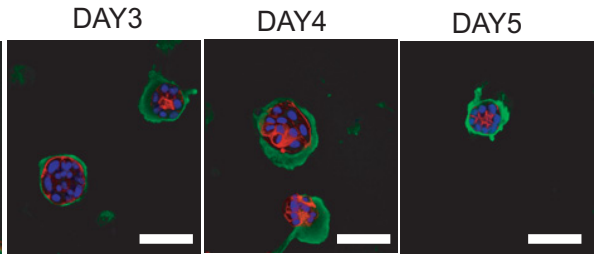

DAY6
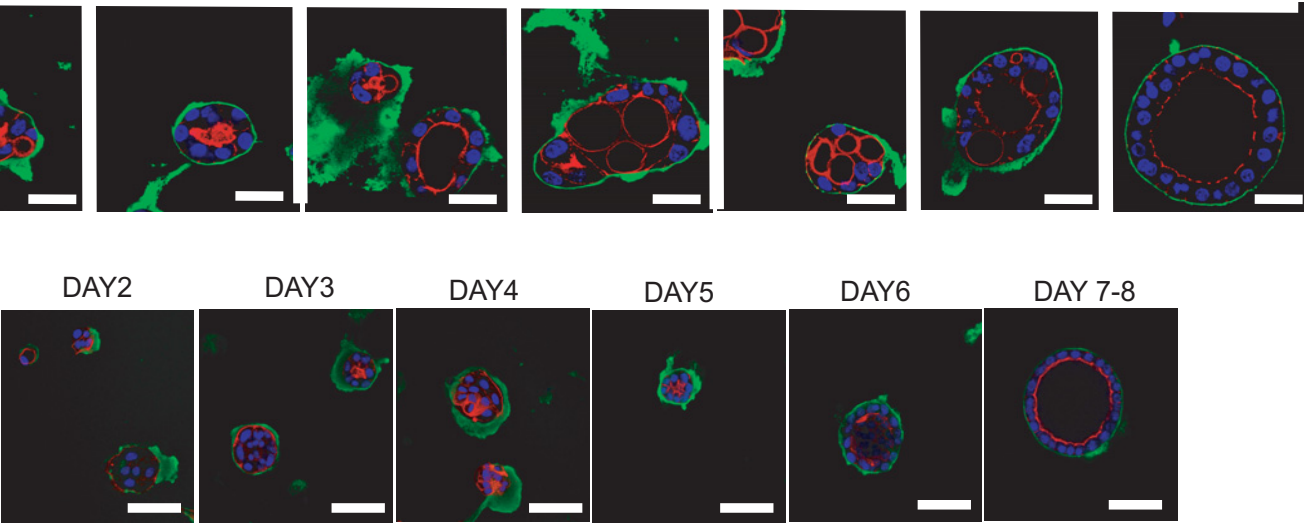

lumen without apototic cells

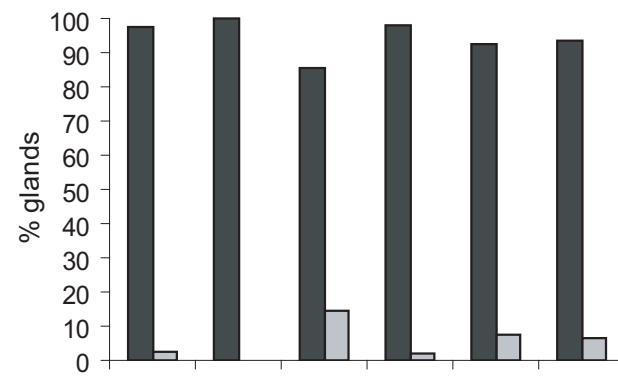

C

口lumen with apoptotic cells

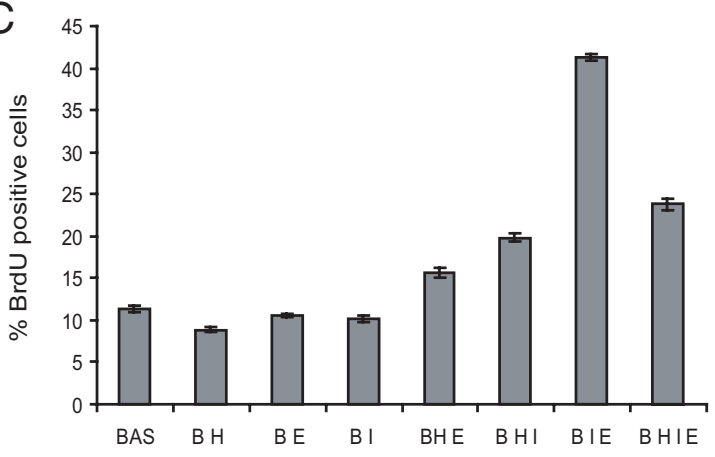

Day 1 Day 2 Day 3 Day 4 Day 5 Day6
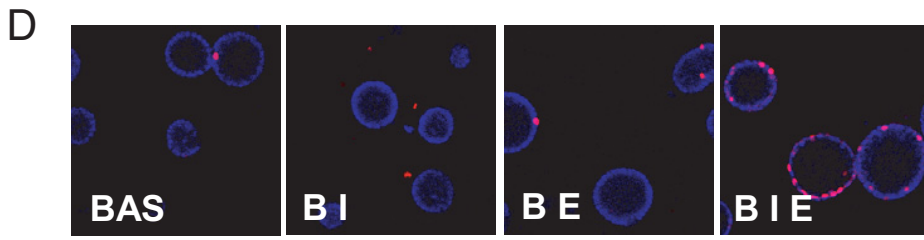

Figure 2. Endometrial epithelial cells develop 3D glandular structures in serum-free defined medium containing insulin and EGF. A: Confocal images of double phalloidin/laminin immunofluorescence of 3D cultures grown in the indicated combinations of medium for 8 days. White scale bar $=20 \mu \mathrm{m}$. B, top: confocal images of phalloidin/laminin staining showing glands growing in BIE medium for the indicated days. Bottom: quantification of nuclei displaying apoptotic morphology in the central part of glands, grown for the indicated days in BIE medium. White scale bar $=20 \mu \mathrm{m}$. C: Quantification of BrdU-positive nuclei in glands growing for 8 days with the indicated combinations of medium culture supplements. D: Representative confocal images showing BrdU incorporation of glands growing in basal medium with the indicated supplement combinations. BAS and B, basal medium; H, hydrocortisone; I, insulin-transferrin-sodium selenite; E, EGF.

cells did not require a bed of gelled Matrigel to develop glandular structures.

The best culture medium for the development of glandular structures was serum-free DMEM/F12 medium containing ITS plus EGF and 3\% diluted Matrigel (Figure 2A). Medium containing either ITS or EGF alone did not allow the formation of 3D polarized glands. In contrast to other
3D culture systems, addition of hydrocortisone to either basal medium or medium containing EGF, ITS, or both factors resulted in the formation of aberrant multiplelumened glands. In medium containing ITS plus EGF (BIE medium), development of single-lumened glands was achieved after 7 to 8 days in culture (Figure 2B). Hoechst staining revealed that most glands did not display cells with 

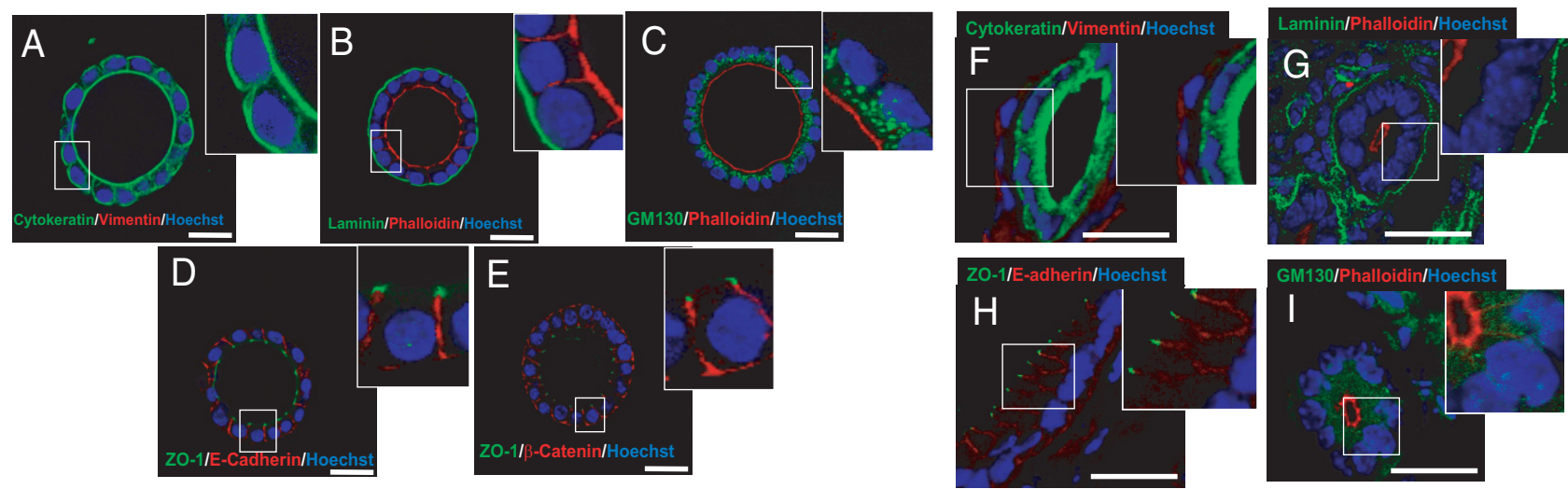

Figure 3. In vitro developed endometrial glands display epithelial apicobasal polarity. Double immunostaining of either in vitro developed glands cultured for 8 days in BIE (A-E) or cryostat sections obtained from mouse endometrium (F-I). A and F: Cytokeratin (green) and vimentin (red). B and G: Laminin (green) and phalloidin (red). C and H: GM130 (green) and phalloidin (red). D and I: ZO-1 (green) and E-cadherin (red). E: ZO-1 (green) and $\beta$-catenin (red). White scale bar $=20 \mu \mathrm{m}$.

typical apoptotic morphology, and the majority of glands displayed a well formed and single central lumen. This result suggests that under these culture conditions, hollowing is the main mechanism of lumen formation (Figure 2B).

The growth-supporting activity of EGF- and ITS-supplemented medium was further assessed by BrdU incorporation (Figure 2C). 3D endometrial cultures were maintained with combinations of EGF, ITS, and hydrocortisone for 7 days. BrdU staining revealed that ITS plus EGF promoted a significant increase of proliferation over all other medium combinations tested (Figure 2, C and D). Medium containing ITS, EGF, and hydrocortisone also promoted a good rate of proliferation but, as we mentioned above, resulted in aberrant acquisition of cell polarity and formation of multiple-lumened glands. For that reason, we chose BIE medium, without hydrocortisone or serum, to culture endometrial glands.

\section{In Vitro Developed Endometrial Glands Display Characteristic Epithelial Apicobasal Polarity}

Once culture conditions were optimized, we analyzed whether the glandular structures displayed correct apicobasal polarization, with an apical side facing a single central lumen, a lateral side containing cell-to-cell contacts, and a basal side with cell-to-extracellular matrix adhesions. To address this point, we cultured endometrial epithelial cells in BIE medium for 7 to 8 days, and we performed immunofluorescence staining for different polarity markers. The endometrial glands developed in our in vitro culture system conserved cytokeratin expression and were completely negative for vimentin, indicating that they were composed exclusively of cells of epithelial origin (Figure $3 \mathrm{~A}$ ). Laminin displayed strong staining on the basal surface surrounding the glandular structures, indicating that the basal surface of cells was "seeding" on rBM. Staining of glands with phalloidin indicated correct positioning of the actin cytoskeleton, mostly located on the apical face of the epithelial cells (Figure 3, B and C). The Golgi apparatus marker GM130 indicated correct positioning of the Golgi apparatus, just above the nucleus and facing the apical surface (Figure 3C). E-cadherin and $\beta$-catenin staining was confined to the lateral side of the epithelial cells, where adherent junctions are located (Figure 3, D and E). Positive immunofluorescence for ZO-1 was located in the limit between lateral and apical surfaces, indicating the correct positioning of tight junctions (Figure 3, D and E). Moreover, 3D reconstruction of glands after immunostaining with phalloidin (red) and E-cadherin (green) demonstrated the presence of glandular structures containing one single lumen, surrounded by polarized epithelial cells all along the glands (Supplemental Video 1, see http://ajp.amjpathol.org).

To demonstrate that epithelial cells displayed organization comparable to that of in vivo endometrium, we performed immunofluorescence staining of cryostat sections obtained from intact uterine tissue. As for in vitro glands (Figure 3A), uterine endometrial epithelial cells were cytokeratin-positive, whereas vimentin staining was restricted to stromal cells (Figure 3F). Laminin and phalloidin staining of the basal lamina and apical actin cytoskeleton observed in Figure 3B was also similar to the in vivo distribution (Figure 3G). ZO-1 and E-cadherin immunostaining revealed similar positioning of adherent and tight junctions in in vitro developed glands (Figure 3D) and in intact endometrium (Figure $3 \mathrm{H})$. GM130 was also located facing apical membrane and above the nucleus (Figure 3l).

All of the above results confirmed that glandular structures displayed the characteristic apicobasal polarity of glandular tissues, as well as the appropriate formation of cell-to-cell and cell-to-extracellular matrix contacts, with patterns similar to those observed in intact endometrium.

\section{Phosphatidylinositol 3-Kinase/Akt Signaling Is Critical for in Vitro Growth of Endometrial Glands}

Having elucidated that EGF and insulin were critical for stimulation of endometrial epithelial proliferation to develop glandular structures, we investigated the signaling pathways used by EGF and insulin to support epithelial cell proliferation and glandular growth. One of the most important pathways in the control of cell proliferation is phosphatidylinositol 3-kinase (PI3K)/Akt, which is very important in endometrial cell homoeostasis. PI3K catalyzes 
A
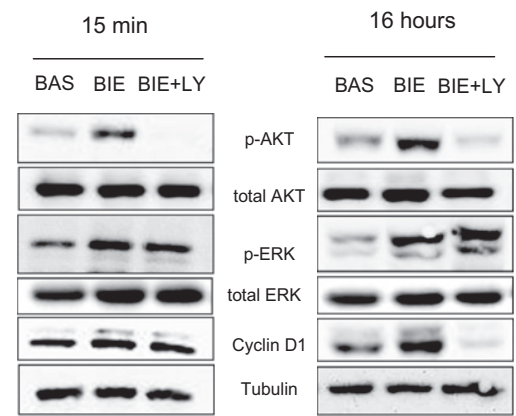

B

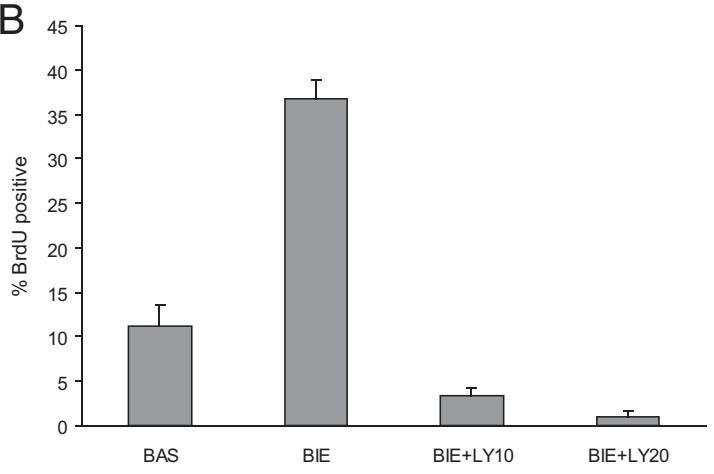

Figure 4. In vitro growth of endometrial glands requires PI3K/Akt signaling A: Western blot analysis showing phosphorylated ERK and Akt and cyclinD1 expression in endometrial glands stimulated 15 minutes or 16 hours with basal medium (BAS), BIE medium, or BIE medium plus $20 \mu \mathrm{mol} / \mathrm{L}$ LY294002 (LY). Membranes were reprobed to ensure equal protein loading. B: Quantification of BrdU-positive nuclei in glands growing in basal medium, BIE medium, or BIE medium plus 10 (LY10) or $20 \mu \mathrm{mol} / \mathrm{L}$ LY294002 (LY20).

the production of phosphatidylinositol-3,4,5-triphosphate by phosphorylating phosphatidylinositol-4,5-diphosphate. One of the key effectors of PI3K signaling is the protein kinase Akt, which is able to recognize this lipid and bind it selectively. Once activated, Akt phosphorylates a range of effector proteins. Among them, GSK-3 is a critical downstream element of the PI3K/Akt pathway whose activity can be inhibited by Akt-mediated phosphorylation at Ser-21 of GSK-3 $\alpha$ and Ser-9 of GSK-3. PTEN phosphatase antagonizes PI3K signaling by dephosphorylating the 3-position of the inositol ring of PI3K and thus inactivating downstream signaling. ${ }^{29}$

For these reasons, we first analyzed the requirement of PI3K/Akt signaling for proliferation of polarized endometrial cells. 3D cultures were grown for 7 days with BIE medium. To return growth factor signaling pathways to the basal state, glands were cultured in basal medium without growth factors for an additional 48 hours. Afterward, glands were stimulated for 15 minutes or 16 hours with basal medium, BIE medium, or BIE medium plus LY294002. Then, Akt, ERK phosphorylation, and cyclinD1 expression were assessed. Stimulation with BIE medium induced phosphorylation of both Akt and ERK at 15 minutes after stimulation, and such phosphorylation was maintained after 16 hours. Consistent with its proliferative effects on glandular structures, BIE medium caused a marked increase in cyclinD1 expression observed after 16 hours of stimulation. Addition of LY294002 to BIE medium caused a drastic decrease of Akt phosphorylation and cyclinD1 expression without affecting phosphorylation of ERK (Figure 4A). Consistent with cy-
clinD1 expression, BIE medium caused a marked increase in BrdU incorporation, which was completely inhibited by addition of LY294002 (Figure 4B). All of the above results suggest that proliferation of polarized glandular cells into the glands depends on Akt signaling.

\section{Down-Regulation of E-Cadherin Disrupts 3D Glandular Structures}

Finally, we investigated whether our 3D culture system is a good tool to study the role of genes controlling glandular morphology in endometrial carcinogenesis. Downregulation of E-cadherin is frequently observed in endometrial carcinogenesis and is associated with disruption of glandular morphology. To mimic E-cadherin loss in endometrial carcinoma, we designed two different shRNAs targeting E-cadherin. Endometrial epithelial cells were infected with lentiviruses carrying E-cadherin shRNAs. Three days after infection, cells were lysed and E-cadherin expression was evaluated by Western blot. Both shRNAs caused a marked down-regulation of Ecadherin protein compared with cells infected with control vector (Figure 5A). We selected shRNA2 for further experiments. To assess the effect of E-cadherin downregulation in glandular architecture, freshly isolated cells were plated in M24 plates and infected with lentiviruses carrying E-cadherin shRNA2 or the empty vector (FSV). After 1 day, cells were trypsinized and cultured in 3D cultures. After 7 to 8 days 3D cultures were fixed and immunostained for E-cadherin and cytokeratin. In cultures infected with E-cadherin shRNA, cells were unable to form glandular structures, and they grew as if they were in a $2 \mathrm{D}$ monolayer (Figure 5B). In contrast, cells infected with the empty vector developed normal glands. To further assess the effects of E-cadherin down-regulation on epithelial organization and morphology, we performed $\beta$-catenin, GM130, and phalloidin immunofluorescence staining on cells with down-regulated E-cadherin. In the absence of E-cadherin, $\beta$-catenin translocated from the membrane to a cytoplasmic/nuclear localization, a phenomenon related to acquisition of a malignant/invasive phenotype (Figure 5B). Moreover, GM130 immunostaining revealed loss of the typical apical localization of the Golgi apparatus, indicating a loss of apicobasal cell polarization. Finally, phalloidin staining indicated actin cytoskeleton reorganization and the formation of actin stress fibers (Figure 5B).

\section{PTEN Down-Regulation Increases Proliferation of 3D Glandular Structures}

Loss of PTEN is one of the most common molecular alterations found in endometrial carcinogenesis. PTEN deficiency leads to increased proliferation in many cell types. To demonstrate that our culture system may be useful to reproduce these alterations, we infected epithelial cells with lentiviruses carrying PTEN shRNA. After 7 to 8 days, glands were deprived of growth factors for 2 days and lysed or assayed for BrdU incorporation. PTEN down-regulation resulted in increased phosphorylation of 
A

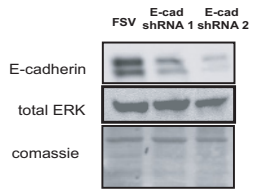

B

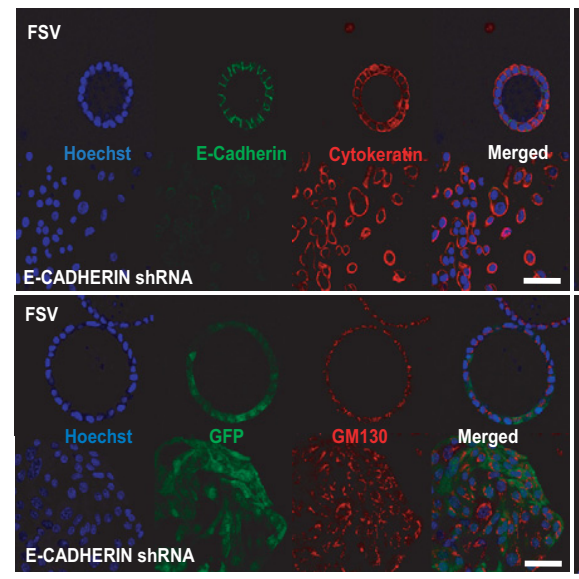

C

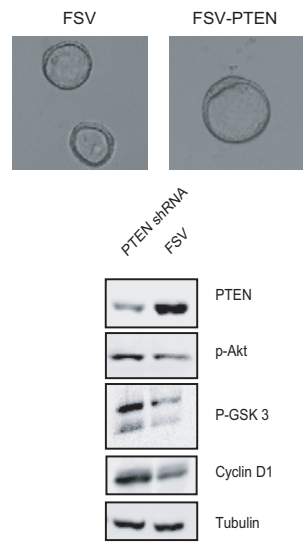

FSV

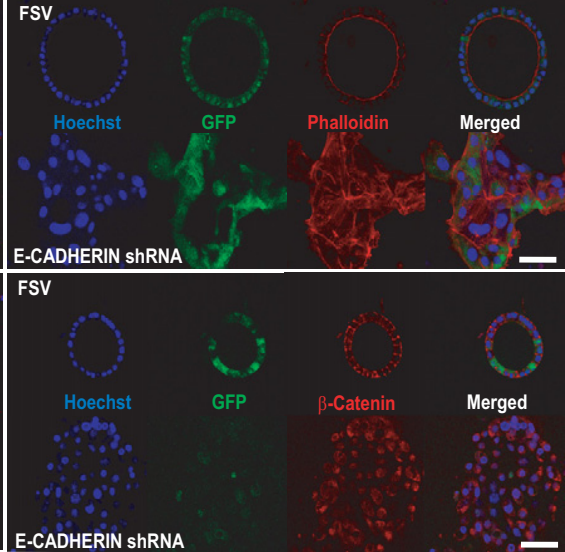

E-CADHERIN ShRNA

Figure 5. E-cadherin knockdown disrupts glandular structures. A: Western blot analysis of glands infected with lentiviruses carrying E-cadherin (E-cad) shRNAs 1 and 2 or the empty vector (FSV). The antibody recognizes two bands corresponding to precursor and mature E-cadherin. Both forms are targeted by the specific shRNAs. Membranes were reprobed with total ERK and stained with Coomassie Blue to ensure equal protein loading. B, top left: Double E-cadherin (green) and cytokeratin (red) immunofluorescence showing down-regulation of E-cadherin expression and disruption of 3D glandular morphology in cells infected with Ecadherin shRNA. Bottom left: Immunofluorescence showing loss of GM130 location in cells infected with E-cadherin shRNA. Top right: Immunofluorescence showing formation of actin stress fibers revealed by phalloidin E-cadherin shRNA-infected cells. Bottom right: Immunofluorescence showing $\beta$-catenin cytoplasmic and nuclear translocation in shRNA-infected cells. Green fluorescent protein (GFP) micrographs were captured before fixation to avoid loss of fluorescence caused by fixation and permeabilization procedures. White scale bar $=20$ $\mu \mathrm{m}$. C, top left: Representative phase-contrast pictures of glands infected with Ientiviruses carrying PTEN shRNA (FSV-PTEN) or the empty vector (FSV). Bottom left: Western blot showing down-regulation of PTEN and increased phospho (p)-Akt, p-GSK3, and cyclinD1 expression of glands infected with lentiviruses carrying PTEN shRNA or the empty vector (FSV). Right: quantification and representative micrographs of BrdU-positive cells in FSV and PTEN shRNAtransfected cells.

Akt and GSK-3 (Figure 5C). We did not observe any difference in the gross morphology of glands infected with PTEN shRNA or the empty vector. However, PTEN knockdown caused a marked increase in cyclin D1 expression, suggesting increased proliferation of PTEN-silenced glands. Consistent with increased cyclin D1 expression, BrdU incorporation revealed a significant proliferation increase in glands with downregulated PTEN (Figure 5C).

\section{Discussion}

There is no doubt that development of 3D cultures represents a valuable tool for understanding the pathogenesis of cancer. ${ }^{21,25,30,31} 3 \mathrm{D}$ cultures mimic the normal phenotype of epithelial cells in vivo and provide a functional and structural environment to investigate the activities of cancer genes. Here, we have developed a novel 3D culture model of primary mouse endometrial epithelial cells. Although cultures of polarized cells from endometrial and other gynecological tissues have been developed previously, ${ }^{32}$ we think that our culture system may have some important innovations that provide several improvements over other existing models. Most 3D cultures are constructed using nontransformed but immortalized cell lines such as MDCK or MCF-10. Here, we culture primary normal and nonimmortalized epithelial cells. This feature may be important to study the processes involved in acquisition of a malignant phenotype, overcoming possible effects derived from cell line immortalization. Moreover, the availability of mouse cells gives the opportunity to examine the effects of mutant mice on gland formation and maintenance of gland structure.

So far, three main systems to develop glandular structures using rBM have been reported: total embedding in rBM; seeding cells on a bed of $100 \%$ gelled rBM plus $2 \%$ rBM diluted in medium; and seeding cells on polyacrylamide-linked rBM with overlaying of diluted rBM. ${ }^{25}$ Here, we propose a new system for developing glandular structures using rBM (Matrigel). We simply resuspend our cells in medium containing $3 \%$ diluted Matrigel and directly plate them into dishes without the layer of $100 \%$ gelled Matrigel. This method markedly decreases the use 
of expensive Matrigel and simplifies the experimental work. These features make our 3D culture inexpensive, fast, and easy to setup and maintain, allowing highthroughput or screening experiments. Moreover, this culture model allows the formation of glandular structures in serum-free defined medium containing only EGF and insulin as growth factors. Matrigel has a poorly defined composition. Therefore, it would be reasonable to think that Matrigel may contain growth factors that induce glandular proliferation and growth. However, we demonstrate that cells grown in basal medium containing 3\% Matrigel but no EGF or insulin fail to develop glandular structures, suggesting that, although Matrigel is absolutely required for establishment of cellular polarization and gland formation, it does not support growth of endometrial glands. The fact that epithelial cells can be maintained in a defined medium in the absence of other cell types such as supporting stromal cells, gives the chance to analyze direct effects of growth factors, hormones, or drugs without the interference of other factors secreted by the other cell populations.

During formation of glands, epithelial cells have to surround a single central lumen with their apical surface facing on it. Two mechanisms of lumen formation have been reported: hollowing and cavitation. ${ }^{1,23,33,34}$ In the first one, lumen is formed by membrane separation of glandular cells; in the second mechanism, lumen forms by death of central cells by either apoptosis ${ }^{35,36}$ or autophagy. ${ }^{37-40}$ Under the growing conditions that we use to develop endometrial glands, hollowing seems to be the main mechanism of lumen formation.

We also demonstrate that growth of endometrial glands triggered by insulin plus EGF combination in vitro depends on PI3K/Akt. PI3K/Akt signaling is critical in maintenance of endometrial homeostasis. Moreover, mutations in PTEN or PIK3CA genes are associated with development and progression of endometrial carcinoma. ${ }^{10,41-47}$ The role of PI3K/ Akt signaling in endometrial carcinogenesis is supported by results obtained in hemizygous PTEN mice ${ }^{48,49}$ and, more recently, by conditional deletion of both copies of PTEN in the endometrium ${ }^{50}$. Female mice lacking a single copy of the PTEN gene develop endometrial hyperplasia, of which approximately $30 \%$ progresses to endometrial carcinoma. The involvement of Akt as a downstream signaling effector of PTEN deficiency has been strongly supported by crossing PTEN hemizygous mice with mice carrying genetic mutations in PTEN downstream effectors. Deficiency in either PDK $1^{51}$ or Akt- $1^{52}$ signaling inhibits tumor development in PTEN hemizygous mice. Similar results have been reported after pharmacological mammalian target of rapamycin inhibition in PTEN-deficient mice. ${ }^{53}$

Epithelial endometrial cells are highly refractory to lipofection (data not shown), but they can be easily infected with lentiviruses, allowing modification of the expression of specific genes by shRNAs or by overexpression experiments. Using this approach, we infected endometrial epithelial cells with PTEN or E-cadherin shRNA. In agreement with the role of alterations of PI3K/Akt pathway in development of endometrial carcinogenesis, knockdown of PTEN caused increased BrdU incorporation and cyclinD1 expression, validating $3 \mathrm{D}$ cultures for the study of proliferative disorders associated with tumor formation.
In epithelial tissues, alterations in cell polarity proteins or proteins involved on adherent or tight junction formation results in increased tumorigenic capacity. E-cadherin is critical in the establishment of adherent junctions between epithelial cells. It is well know that down-regulation of Ecadherin expression is associated with tumoral progression in a wide variety of carcinomas in different locations. ${ }^{12,54}$ E-cadherin is a calcium-dependent adhesion molecule. The extracellular regions are responsible for adhesive recognition and binding other cadherins presented on neighboring cells. On the other side of the plasma membrane, the cadherin cytoplasmic tails interact with several proteins that link the cadherin receptor to the actin cytoskeleton. The best understood of these associated proteins is $\beta$-catenin, which binds directly to the distal region of the cytoplasmic tail. $\beta$-Catenin, in addition to its function in this cell adhesion complex, acts as a key element in the Wnt signal transduction pathway, which has been implicated in embryogenesis and carcinogenesis. ${ }^{55,56}$ Therefore, loss of E-cadherin expression results in a cytosolic accumulation of $\beta$-catenin, leading to its complex formation with transcription factors such as Tcf/Lef-1, translocation into the nucleus, and induction of transcription of responsive genes involved in the acquisition of the tumoral phenotype. ${ }^{2,12,56}$ Moreover, loss of adherent junctions after down-regulation of E-cadherin expression results in loss of cell polarity, which leads to release of the Scrib complex, Par proteins, and Rho GTPases from cell-to-cell contacts to promote migration. ${ }^{3,4}$ In line with these results, here we have demonstrated that the down-regulation of E-cadherin is sufficient to cause a complete loss of cell polarity accompanied by the acquisition of features associated with a malignant/migratory phenotype such as $\beta$-catenin translocation, Golgi delocalization, and polymerization of stress fibers.

In summary, we described here a new 3D system to culture endometrial cells of murine origin. This culture may provide a valuable tool to study morphological changes in endometrial glandular architecture associated with endometrial carcinogenesis.

\section{References}

1. Bryant DM, Mostov KE: From cells to organs: building polarized tissue. Nat Rev Mol Cell Biol 2008, 9:887-901

2. Lee M, Vasioukhin V: Cell polarity and cancer-cell and tissue polarity as a non-canonical tumor suppressor. J Cell Sci 2008, 121:1141-1150

3. Etienne-Manneville S: Polarity proteins in migration and invasion. Oncogene 2008, 27:6970-6980

4. Tanos B, Rodriguez-Boulan E: The epithelial polarity program: machineries involved and their hijacking by cancer. Oncogene 2008, 27:6939-6957

5. Wodarz A, Nathke I: Cell polarity in development and cancer. Nat Cell Biol 2007, 9:1016-1024

6. Hedrick L, Cho KR, Vogelstein B: Cell adhesion molecules as tumour suppressors. Trends Cell Biol 1993, 3:36-39

7. Cowin P, Rowlands TM, Hatsell SJ: Cadherins and catenins in breast cancer. Curr Opin Cell Biol 2005, 17:499-508

8. Palacios J, Robles-Frias MJ, Castilla MA, Lopez-Garcia MA, Benitez $\mathrm{J}$ : The molecular pathology of hereditary breast cancer. Pathobiology 2008, 75:85-94

9. Schlosshauer PW, Ellenson LH, Soslow RA: $\beta$-Catenin and E-cadherin expression patterns in high-grade endometrial carcinoma are associated with histological subtype. Mod Pathol 2002, 15:1032-1037

10. Llobet D, Pallares J, Yeramian A, Santacana M, Eritja N, Velasco A, 
Dolcet X, Matias-Guiu X: Molecular pathology of endometrial carcinoma: practical aspects from the diagnostic and therapeutic viewpoints. J Clin Pathol 2009, 62:777-785

11. Moreno-Bueno G, Hardisson D, Sarrio D, Sanchez C, Cassia R, Prat J, Herman JG, Esteller M, Matias-Guiu X, Palacios J: Abnormalities of $\mathrm{E}-$ and P-cadherin and catenin ( $\beta-, \gamma$-catenin, and p120ctn) expression in endometrial cancer and endometrial atypical hyperplasia. J Pathol 2003, 199:471-478

12. Jeanes A, Gottardi CJ, Yap AS: Cadherins and cancer: how does cadherin dysfunction promote tumor progression? Oncogene 2008, 27:6920-6929

13. Moreno-Bueno G, Portillo F, Cano A: Transcriptional regulation of cell polarity in EMT and cancer. Oncogene 2008, 27:6958-6969

14. Michalopoulos G, Pitot HC: Primary culture of parenchymal liver cells on collagen membranes. Morphological and biochemical observations. Exp Cell Res 1975, 94:70-78

15. Emerman JT, Burwen SJ, Pitelka DR: Substrate properties influencing ultrastructural differentiation of mammary epithelial cells in culture. Tissue Cell 1979, 11:109-119

16. Emerman JT, Pitelka DR: Maintenance and induction of morphological differentiation in dissociated mammary epithelium on floating collagen membranes. In Vitro 1977, 13:316-328

17. Elsdale T, Bard J: Collagen substrata for studies on cell behavior J Cell Biol 1972, 54:626-637

18. Hall HG, Farson DA, Bissell MJ: Lumen formation by epithelial cell lines in response to collagen overlay: a morphogenetic model in culture. Proc Natl Acad Sci USA 1982, 79:4672-4676

19. Kim JB, Stein R, O'Hare MJ: Three-dimensional in vitro tissue culture models of breast cancer-a review. Breast Cancer Res Treat 2004, 85:281-291

20. Hebner C, Weaver VM, Debnath J: Modeling morphogenesis and oncogenesis in three-dimensional breast epithelial cultures. Annu Rev Pathol 2008, 3:313-339

21. Kim JB: Three-dimensional tissue culture models in cancer biology Semin Cancer Biol 2005, 15:365-377

22. Debnath J, Muthuswamy SK, Brugge JS: Morphogenesis and oncogenesis of MCF-10A mammary epithelial acini grown in three-dimensional basement membrane cultures. Methods 2003, 30:256-268

23. Mailleux AA, Overholtzer M, Brugge JS: Lumen formation during mammary epithelial morphogenesis: insights from in vitro and in vivo models. Cell Cycle 2008, 7:57-62

24. Shaw KR, Wrobel CN, Brugge JS: Use of three-dimensional basement membrane cultures to model oncogene-induced changes in mammary epithelial morphogenesis. J Mammary Gland Biol Neoplasia 2004, 9:297-310

25. Debnath J, Brugge JS: Modelling glandular epithelial cancers in three-dimensional cultures. Nat Rev Cancer 2005, 5:675-688

26. Bigsby RM, Cooke PS, Cunha GR: A simple efficient method for separating murine uterine epithelial and mesenchymal cells. Am J Physiol 1986, 251:E630-E636

27. Pallares J, Llobet D, Santacana M, Eritja N, Velasco A, Cuevas D, Lopez S, Palomar-Asenjo V, Yeramian A, DolcetX, Matias-Guiu X: CK2 $\beta$ is expressed in endometrial carcinoma and has a role in apoptosis resistance and cell proliferation. Am J Pathol 2009, 174:287-296

28. Dolcet X, Llobet D, Encinas M, Pallares J, Cabero A, Schoenenberger JA, Comella JX, Matias-Guiu X: Proteasome inhibitors induce death but activate $\mathrm{NF}-\kappa \mathrm{B}$ on endometrial carcinoma cell lines and primary culture explants. J Biol Chem 2006, 281:22118-22130

29. Sansal I, Sellers WR: The biology and clinical relevance of the PTEN tumor suppressor pathway. J Clin Oncol 2004, 22:2954-2963

30. Fischbach C, Chen R, Matsumoto T, Schmelzle T, Brugge JS, Polverini PJ, Mooney DJ: Engineering tumors with 3D scaffolds. Nat Methods 2007, 4:855-860

31. Yamada KM, Cukierman E: Modeling tissue morphogenesis and cancer in 3D. Cell 2007, 130:601-610

32. Adissu HA, Asem EK, Lelievre SA: Three-dimensional cell culture to model epithelia in the female reproductive system. Reprod Sci 2007, 14:11-19

33. Martin-Belmonte F, Mostov K: Regulation of cell polarity during epithelial morphogenesis. Curr Opin Cell Biol 2008, 20:227-234

34. Martín-Belmonte F, Yu W, Rodriguez-Fraticelli AE, Ewald AJ, Werb Z Alonso MA, Mostov K: Cell-polarity dynamics controls the mechanism of lumen formation in epithelial morphogenesis. Curr Biol 2008, 18:507-513
35. Debnath J, Mills KR, Collins NL, Reginato MJ, Muthuswamy SK, Brugge JS: The role of apoptosis in creating and maintaining luminal space within normal and oncogene-expressing mammary acini. Cell 2002, 111:29-40

36. Mailleux AA, Overholtzer M, Schmelzle T, Bouillet $P$, Strasser A, Brugge JS: BIM regulates apoptosis during mammary ductal morphogenesis, and its absence reveals alternative cell death mechanisms. Dev Cell 2007, 12:221-234

37. Debnath J: Detachment-induced autophagy during anoikis and lumen formation in epithelial acini. Autophagy 2008, 4:351-353

38. Debnath J: Detachment-induced autophagy in three-dimensional epithelial cell cultures. Methods Enzymol 2009, 452:423-439

39. Lock R, Debnath J: Extracellular matrix regulation of autophagy. Curr Opin Cell Biol 2008, 20:583-588

40. Mills KR, Reginato M, Debnath J, Queenan B, Brugge JS: Tumor necrosis factor-related apoptosis-inducing ligand (TRAIL) is required for induction of autophagy during lumen formation in vitro. Proc Nat Acad Sci USA 2004, 101:3438-3443

41. Levine RL, Cargile CB, Blazes MS, van Rees B, Kurman RJ, Ellenson LH: PTEN mutations and microsatellite instability in complex atypical hyperplasia, a precursor lesion to uterine endometrioid carcinoma Cancer Res 1998, 58:3254-3258

42. Tashiro H, Blazes MS, Wu R, Cho KR, Bose S, Wang SI, Li J, Parsons $\mathrm{R}$, Ellenson LH: Mutations in PTEN are frequent in endometrial carcinoma but rare in other common gynecological malignancies. Cancer Res 1997, 57:3935-3940

43. Bussaglia E, del Rio E, Matias-Guiu X, Prat J: PTEN mutations in endometrial carcinomas: a molecular and clinicopathologic analysis of 38 cases. Hum Pathol 2000, 31:312-317

44. Matias-Guiu X, Catasus L, Bussaglia E, Lagarda H, Garcia A, Pons C Munoz J, Arguelles R, Machin P, Prat J: Molecular pathology of endometrial hyperplasia and carcinoma. Hum Pathol 2001, 32:569-577

45. Velasco A, Bussaglia E, Pallares J, Dolcet X, Llobet D, Encinas M Llecha N, Palacios J, Prat J, Matias-Guiu X: PIK3CA gene mutations in endometrial carcinoma: correlation with PTEN and K-RAS alterations. Hum Pathol 2006, 37:1465-1472

46. Hayes MP, Douglas W, Ellenson LH: Molecular alterations of EGFR and PIK3CA in uterine serous carcinoma. Gynecol Oncol 2009, 113:370-373

47. Hayes MP, Wang H, Espinal-Witter R, Douglas W, Solomon GJ, Baker SJ, Ellenson LH: PIK3CA and PTEN mutations in uterine endometrioid carcinoma and complex atypical hyperplasia. Clin Cancer Res 2006, 12:5932-5935

48. Di Cristofano A, Pesce B, Cordon-Cardo C, Pandolfi PP: Pten is essential for embryonic development and tumour suppression. Nat Genet 1998, 19:348-355

49. Podsypanina K, Ellenson LH, Nemes A, Gu J, Tamura M, Yamada KM, Cordon-Cardo C, Catoretti G, Fisher PE, Parsons R: Mutation of Pten/Mmac1 in mice causes neoplasia in multiple organ systems. Proc Natl Acad Sci USA 1999, 96:1563-1568

50. Daikoku T, Hirota Y, Tranguch S, Joshi AR, DeMayo FJ, Lydon JP, Ellenson LH, Dey SK: Conditional loss of uterine Pten unfailingly and rapidly induces endometrial cancer in mice. Cancer Res 2008, 68:5619-5627

51. Bayascas JR, Leslie NR, Parsons R, Fleming S, Alessi DR: Hypomorphic mutation of PDK1 suppresses tumorigenesis in $\mathrm{PTEN}{ }^{+/-}$mice. Curr Biol 2005, 15:1839-1846

52. Chen ML, Xu PZ, Peng XD, Chen WS, Guzman G, Yang X, Di Cristofano A, Pandolfi PP, Hay N: The deficiency of Akt1 is sufficient to suppress tumor development in $\mathrm{Pten}^{+/-}$mice. Genes Dev 2006, 20:1569-1574

53. Podsypanina K, Lee RT, Politis C, Hennessy I, Crane A, Puc J, Neshat M, Wang H, Yang L, Gibbons J, Frost P, Dreisbach V, Blenis J, Gaciong Z, Fisher P, Sawyers C, Hedrick-Ellenson L, Parsons R: An inhibitor of mTOR reduces neoplasia and normalizes p70/S6 kinase activity in Pten $^{+/-}$mice. Proc Natl Acad Sci USA 2001, 98:10320-10325

54. Perl AK, Wilgenbus P, Dahl U, Semb H, Christofori G: A causal role for E-cadherin in the transition from adenoma to carcinoma. Nature 1998 392:190-193

55. Anastasiadis PZ, Reynolds AB: The p120 catenin family: complex roles in adhesion, signaling and cancer. J Cell Sci 2000, 113(Pt 8):1319-1334

56. Peifer M, Polakis P: Wnt signaling in oncogenesis and embryogenesis-a look outside the nucleus. Science 2000, 287:1606-1609 